\title{
Grade III Spleen Laceration After a Colonoscopy Treated with Splenic Artery Embolization: A Case Report
}

\author{
Mohamed Ahmed ${ }^{1}$, Saba Habis ${ }^{2}$, Rasha Saeed ${ }^{1}$, Ahmed Mahmoud ${ }^{1}$, Scott H. Kwok ${ }^{3}$ \\ 1. Surgery, Riverside Community Hospital, Riverside, USA 2. Internal Medicine, Riverside Community Hospital, \\ Riverside, USA 3. Radiology, Riverside Community Hospital, Riverside, USA
}

Corresponding author: Mohamed Ahmed, maamsmd@yahoo.com

\section{Abstract}

Our patient is a 58-year-old female who presented to our emergency room with left upper quadrant abdominal pain the day after outpatient screening colonoscopy. A computed tomography (CT) scan of the abdomen and pelvis revealed a grade III spleen injury. She was admitted to our intensive care unit, and a gradual decline in her hematocrit was noticed. The patient did well and was discharged from hospital the day after splenic artery embolization.

Categories: Radiology, Gastroenterology, General Surgery

Keywords: colonoscopy, spleen laceration, coil embolization

\section{Introduction}

Colonoscopy is the most common procedure for the diagnosis and treatment of lower gastrointestinal diseases, with fifteen million colonoscopies performed in the United States in 2012 [1]. A screening colonoscopy is a commonly performed outpatient procedure, with minimal morbidity. The reported complications rate averages $0.4 \%$, which most commonly includes bleeding, perforation, and, rarely, splenic rupture [2]. There are just over 100 reported cases of splenic injury following colonoscopy in the literature, and it was first reported by Wherry et al. in 1974 [3]. The diagnosis is confirmed by a computed tomography (CT) scan of the abdomen and pelvis. We report a case of splenic rupture following colonoscopy, which was treated with splenic artery embolization.

\section{Case Presentation}

A 58-year-old female presented to our emergency room with worsening left upper quadrant abdominal pain radiating to her left shoulder the day after outpatient colonoscopy. A CT scan of the abdomen and pelvis revealed a grade III splenic injury with no obvious active extravasation (Figure 1).

Received 12/17/2018

Review began 12/21/2018 Review ended 01/06/2019 Published 01/08/2019

๑) Copyright 2019

Ahmed et al. This is an open access article distributed under the terms of the Creative Commons Attribution License CC-BY 3.0., which permits unrestricted use, distribution, and reproduction in any medium, provided the original author and source are credited. 


\section{Cureus}

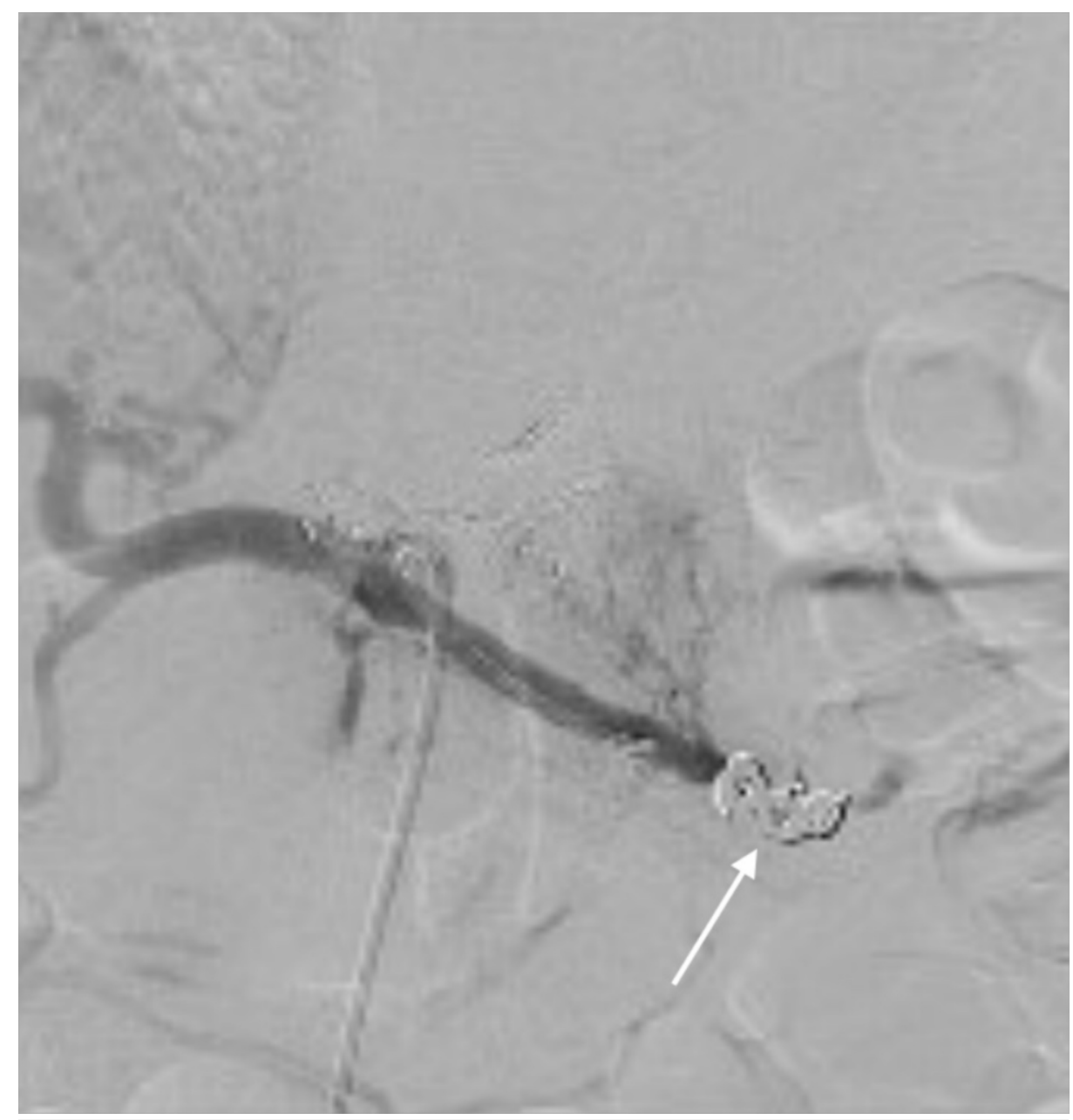

\section{FIGURE 1: Angioembolization}

Splenic artery embolized, with no blood flow distal to the coils. Arrow indicates the interlocking coils

She was admitted to our intensive care unit and her initial hemoglobin ( 9 grams per deciliter) dropped gradually to 7.4 grams per deciliter over 24 hours. Angiography revealed a subcapsular blush (Figure 2). Splenic artery embolization, with interlocking coils, was performed (Figure 3). The patient did well with no further drop in her hemoglobin and was discharged from the hospital the following day. 


\section{Cureus}

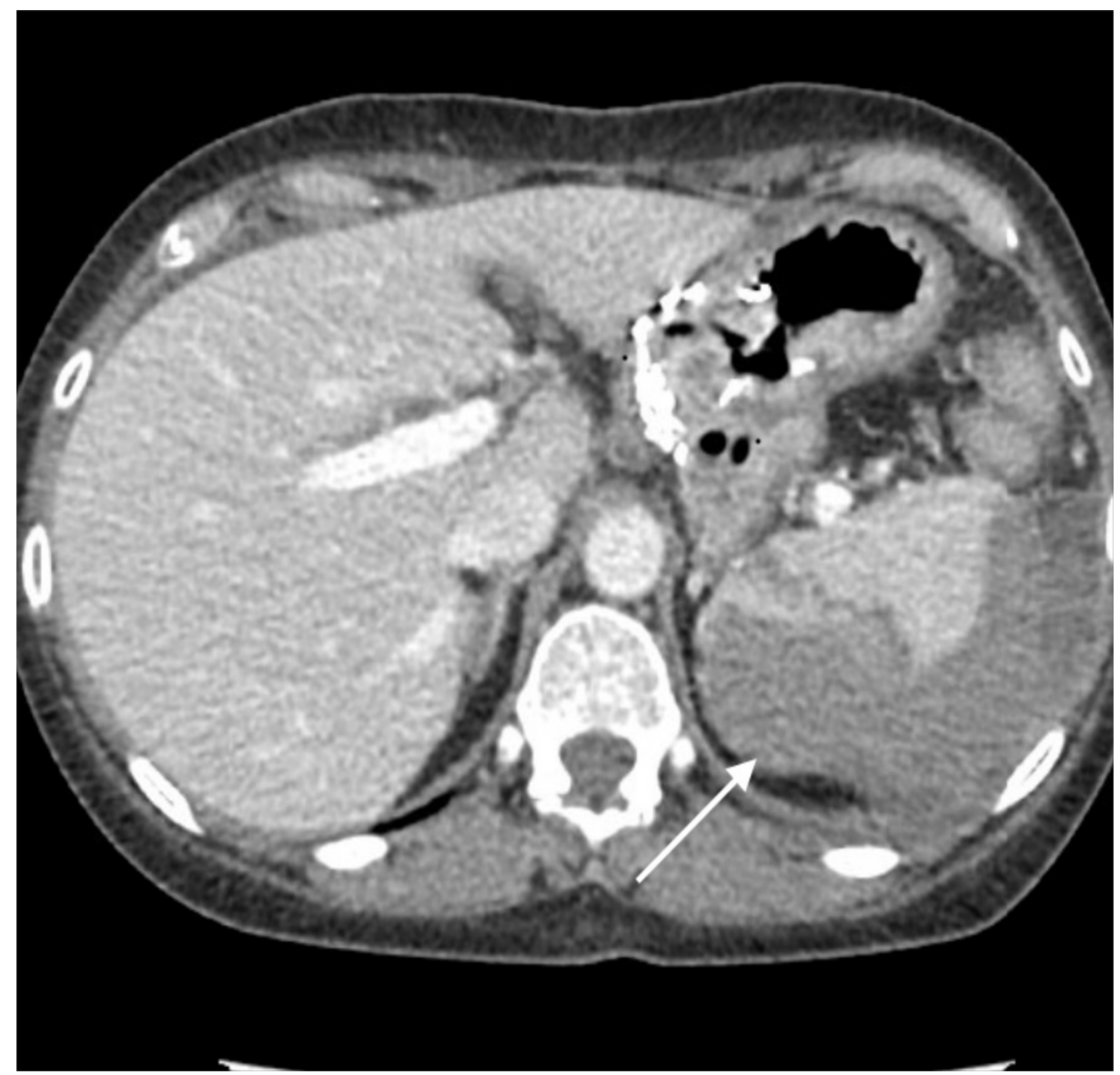

FIGURE 2: Grade III splenic injury with no obvious active extravasation 


\section{Cureus}

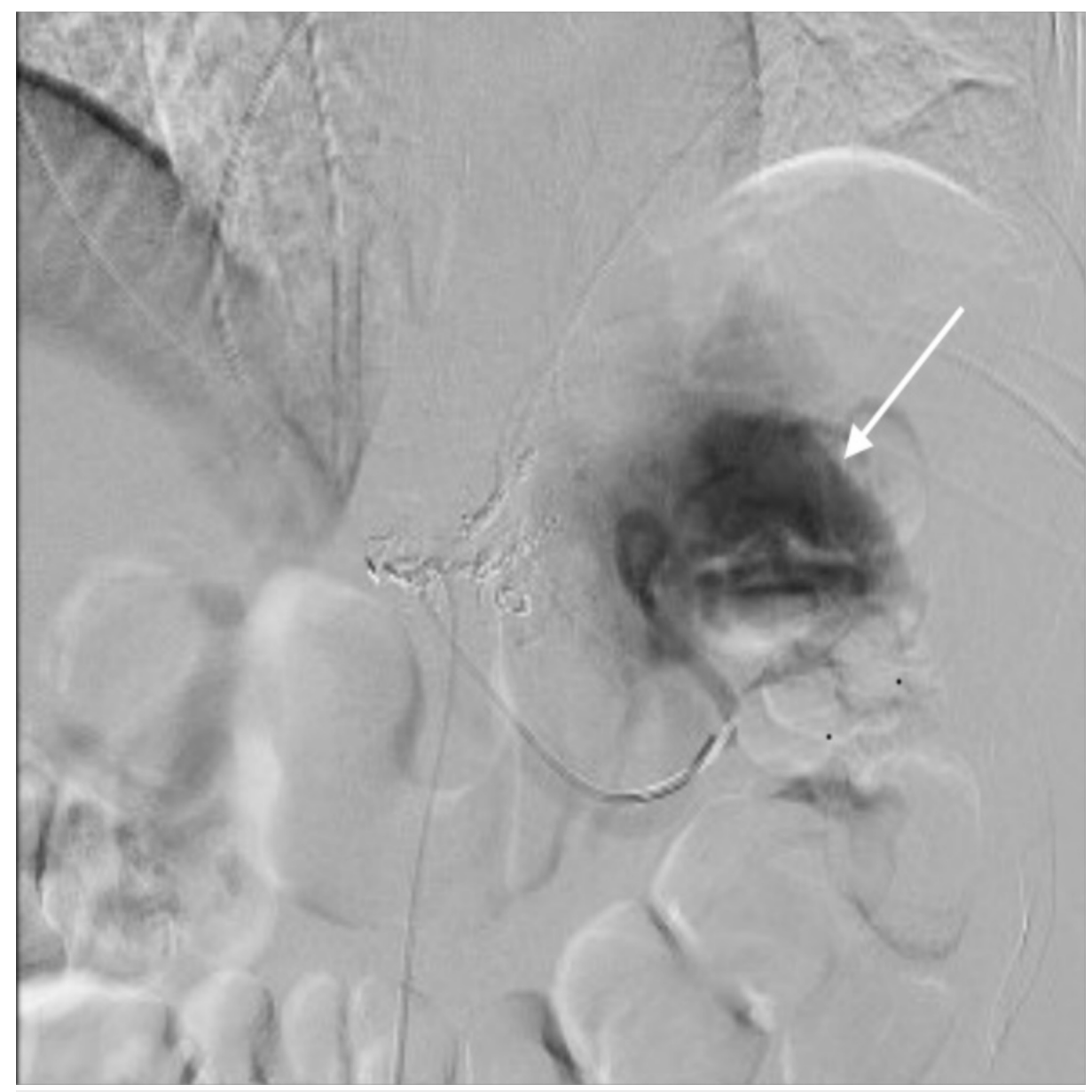

FIGURE 3: Splenic artery angiography

Splenic artery angiography demonstrates active bleeding (blush), which is demonstrated by the white arrow

\section{Discussion}

The number of colonoscopies performed across the United States is increasing, and the potential complication of splenic injury should be on the examining physicians' differential [1]. Seventy-five percent of traumatic spleen injuries are managed conservatively, with good success, which does not appear to be the case in colonoscopy-induced injury. Available data suggest that $75 \%$ of these patients fail conservative management [4]. The majority of cases developed symptoms within 24 hours of the procedure, with abdominal pain being the most common presenting complaint (94\%) and hypotension in $55 \%$ of the cases associated with a significant decrease in hematocrit. The CT scan of the abdomen and pelvis provides the most sensitive and specific method of diagnosis. Predictors of failed conservative management were grade II or above splenic laceration, old age, pre-existing splenic disease, hemodynamic instability, one unit of blood transfusion, hemoperitoneum, and female sex (75\%) [5-6]. Neither a history of abdominal surgery nor the performance of a biopsy seems to increase the incidence of splenic injury [7]. In hemodynamically unstable patients, splenectomy is the definitive management. Nonoperative management was carried out successfully in $22 \%$ of patients who sustained a spleen injury after colonoscopy. Splenic artery embolization, which potentially preserves splenic function, was reported in a few cases [8].

\section{Conclusions}

Splenic injury after colonoscopy is a rare complication and requires a high index of diagnostic suspicion when a patient presents with abdominal pain after colonoscopy associated with hemodynamic instability. While splenic injury after trauma can be managed conservatively in the majority of cases, this does not appear to be the case with a colonoscopy-induced injury. Angioembolization of the splenic artery appears to be very promising for the management of splenic ruptures.

\section{Additional Information \\ Disclosures}

Human subjects: Consent was obtained by all participants in this study. Conflicts of interest: In 
compliance with the ICMJE uniform disclosure form, all authors declare the following: Payment/services info: All authors have declared that no financial support was received from any organization for the submitted work. Financial relationships: All authors have declared that they have no financial relationships at present or within the previous three years with any organizations that might have an interest in the submitted work. Other relationships: All authors have declared that there are no other relationships or activities that could appear to have influenced the submitted work.

\section{References}

1. Joseph DA, Meester RGS, Zauber AG, et al.: Colorectal cancer screening: estimated future colonoscopy need and current volume and capacity. Cancer. 2016, 122:2479-2486. 10.1002/cncr.30070

2. Zwink N, Holleczek B, Stegmaier C, Hoffmeister M, Brenner H: Complication rates in colonoscopy screening for cancer. A prospective cohort study of complications arising during the procedure and in the ensuing four weeks. Dtsch Arztebl Int. 2017, 114:321-327. 10.3238/arztebl.2017.0321

3. Ozogul B, Kisaoglu A, Bayramoglu A, Kara S, Aksungur N: Laparascopic splenectomy due to splenic injury after colonoscopy. Eurasian J Med. 2016, 48:153-155. 10.5152/eurasianjmed.2015.15040

4. Shankar S, Rowe S: Splenic injury after colonoscopy: case report and review of literature . Ochsner J. 2011, 11:276-281.

5. McBride R, Dasari B, Magowan H, Mullan M, Yousaf M, Mackle E: Splenic injury after colonoscopy requiring splenectomy. BMJ Case Rep. 2013, 2013:bcr2013009126. 10.1136/bcr-2013-009126

6. Ng P: Splenic injury as a complication of colonoscopy: more common than we think? . BMJ Case Rep. 2015, 2015:bcr2015209707. 10.1136/bcr-2015-209707

7. Skipworth JR, Raptis DA, Rawal JS, Olde Damink S, Shankar A, Malago M, Imber C: Splenic injury following colonoscopy - an underdiagnosed, but soon to increase, phenomenon?. Ann R Coll Surg Engl. 2009, 91:W611 .

8. Brennan I, Faintuch S, Sacks B: Superselective splenic artery embolization for the management of splenic laceration following colonoscopy. Acta Radiologica Open. 2014, 3:10.1177/2047981614524199 